\title{
Motivators and barriers for paediatricians discharging patients
}

\author{
Erin Turbitt $^{\mathrm{A}, \mathrm{B}}$, Marina Kunin $^{\mathrm{A}}$, Sarah Gafforini ${ }^{\mathrm{A}}$ and Gary L. Freed ${ }^{\mathrm{A}, \mathrm{C}}$ \\ A Health Systems and Workforce Unit, The Centre for Health Policy, School of Population and Global Health, \\ The University of Melbourne, Level 4, 207 Bouverie Street, Parkville, Vic. 3010, Australia. \\ ${ }^{B}$ Present address: National Human Genome Research Institute, Building 31, Room B1B54, 31 Center Drive, \\ Bethesda, MD 20892-2073, USA. \\ ${ }^{\mathrm{C}}$ Corresponding author. Email: gary.freed@unimelb.edu.au
}

\begin{abstract}
The aim of this study was to identify motivators and barriers that paediatricians face when discharging patients from outpatient specialty care. A questionnaire was administered to outpatient care paediatricians in Victoria working in one of five speciality public hospital clinics. Questions focused on how important various motivators and barriers were in respondents' decision to discharge a patient from their clinic. Nearly all $(91 \%, n=74)$ paediatricians invited to participate provided responses. The factor influencing the greatest proportion of paediatricians in their decision to discharge patients back to primary care was the potential that patients may not receive the required care from a GP. The next most highly rated barrier was that it is too complicated to arrange discharge; rated as a very important influence by one-third of paediatricians $(33 \%, n=24)$. Improvements to the discharge process may encourage more paediatricians to discharge patients back to their GP, therefore freeing up appointment slots. This in turn could reduce waiting times for paediatric outpatient clinics in Victoria. The concern from paediatricians that patients may not receive the required care from a GP warrants attention and should be further investigated.
\end{abstract}

Additional keywords: child health services, hospital outpatient clinics, patient discharge, referral and consultation.

Received 5 August 2016, accepted 7 October 2016, published online 30 November 2016

\section{Introduction}

Referral rates to general and sub-speciality paediatricians have increased in Australia in recent years (John Stanway, pers. comm., 2015). Furthermore, low discharge rates have led to long waiting times for paediatric outpatient care, in particular for new patients, with calls for improvements in the 'new to review' patient ratio (Department of Health and Human Services Victoria Australia 2009). In Australia, GPs play a key role in the follow-up and management of children who have received specialist care (Department of Health and Human Services Victoria Australia 2010).

Long waiting times for speciality care may, in part, be a consequence of clinicians' reluctance to discharge patients back to primary care. Previous research suggests many potential reasons for this reluctance: low confidence in GPs to provide follow-up care, the influence of patient expectations and pressures, and additional workload for specialists when arranging discharge (Dodd et al. 1994; Burkey et al. 1997). Recent Australian research found that just one-fifth of review patients received advice from a paediatrician to follow-up with a GP, and $68 \%$ of patients preferred to follow up with a paediatrician (Kunin et al. 2016). With the exception of this one study, other existing research was conducted close to two decades ago. More recent, paediatric-focused data are required to guide policy and practice.
The concept of a medical home has been endorsed by professional organisations, in particular for children with chronic, complex conditions (American Academy of Paediatrics 2002; National Health and Hospitals Reform Commission 2009). The medical home provides patients with a central point of contact (generally a primary care physician) to receive coordinated and continuous care. A medical home that functions efficiently requires specialists to discharge patients back to GPs for follow up and ongoing management, and allows GPs to coordinate care across many providers if necessary. This relies on successful relationships between specialists and GPs.

There is no previous Australian research on the motivators and barriers paediatricians face when discharging patients from outpatient specialty care. Evidence on this topic could inform strategies to reduce waiting times for outpatient specialist paediatrician care through improvements to the discharge process. We aimed to explore this topic in a study investigating the perspectives of paediatricians working in public outpatient speciality clinics in Melbourne, Australia.

\section{Methods}

This report is part of a wider study to investigate multiple aspects of referrals to public outpatient paediatricians in Melbourne, Australia. 


\section{What is known about the topic?}

- Low discharge rates have led to long waiting times for paediatric outpatient care in Australia.

\section{What does this paper add?}

- Victorian paediatricians noted concern that a patient would not receive the required care without their involvement as an important influence in their decision to discharge patients.

\section{Sample and recruitment}

The sample consisted of paediatricians working in one of five paediatric outpatient speciality clinics at the two largest paediatric public hospitals in metropolitan Melbourne. The five specialties were: (i) general paediatrics, (ii) respiratory medicine, (iii) developmental medicine or community child health, (iv) neurology and (v) endocrinology. These five specialties were chosen as they have the highest volume of medical (non-surgical) patients excluding haematology and oncology clinics (which have defined protocols for care) and cardiology clinics (which have a large proportion of post-surgical patients). Recruitment targets at each hospital were $100 \%$ of paediatricians identified by the respective hospitals as practicing in these specialties $(N=83)$. Paediatricians were surveyed during August to October 2015.

At the first mailing of the survey, paediatricians were provided with a co-branded (The University of Melbourne and the two hospitals) information sheet about the study, a questionnaire, a reply paid envelope and $\$ 5$ cash in appreciation for their participation. The information sheet advised paediatricians they would receive up to two reminders and they could notify the research team if they did not want to be contacted again. Paediatricians were informed that returning the anonymous questionnaire would constitute consent to participate. Participants were guaranteed confidentiality and that data reported would be in aggregate form. On initial mailing, the questionnaire was administered in hard copy by registered mail. Two follow-up mailings were sent to non-respondents by regular mail.

\section{Questionnaire}

The questionnaire was developed by the research team in consultation with department heads of each speciality or their delegate at each hospital. Items developed were based on a supplementary literature review about the interface between primary and speciality paediatric care. The questionnaire explored paediatricians' opinions of new referrals from GPs and community paediatricians, shared-care arrangements, follow-up care and patient discharge.

Seven questions from the larger questionnaire were analysed for this report, which related specifically to follow-up care and discharge planning. Five questions asked participants how important various motivators and barriers were in their decision to discharge a patient from their clinic. Responses were given on a four-point Likert scale (unimportant, not very important, somewhat important or very important). Two questions asked about the frequency with which parents had influenced the follow up and discharge decision-making process. Responses were given on a four-point Likert scale consisting of rarely $(<10 \%)$, sometimes (10-50\%), frequently (51-90\%) and almost always (>90\%). Participant characteristics and demographics were also collected (gender, years' experience in speciality and proportion of full-time equivalent worked in their clinic).

The project received ethics approval from The University of Melbourne, and both hospital human research ethics committees.

\section{Data analysis}

Initially, frequency distributions and descriptive analyses were conducted. Next, bivariate analyses using Chi-Square statistics were used to determine the differences, if any, among respondents based on the participant characteristic variables collected. The characteristics examined included speciality (general paediatrics $v$. other), gender, years of experience in speciality, and proportion of full-time equivalent worked in their clinic. We also examined associations between motivators and barriers to discharge from the clinic and the frequency that paediatricians experience when discharging patients from outpatient specialty care. For these analyses, responses were dichotomised as: unimportant or not very important $v$. somewhat important or very important; and rarely $v$. sometimes, frequently or almost always. Data were analysed using STATA 13.0 (StataCorp College Station, TX, USA).

\section{Results}

Responses were received from nearly all $(91 \%, n=74)$ paediatricians working in one of the five selected public paediatric outpatient specialty clinics in Victoria. All non-respondents worked in general paediatric clinics. The sample consisted of slightly more females $(55 \%, n=41)$ than males, with nearly twothirds $(64 \%, n=47)$ having over 10 years' experience in their speciality. Slightly over half of the sample worked less than 0.5 full-time equivalent in the specialist clinic $(55 \%, n=41)$. The speciality most highly represented was general paediatrics $(42 \%, n=32)$, with approximately equal numbers of respondents in the remaining four specialties (Table 1 ).

\section{Motivators and barriers to discharge patients to GP care}

Concern that the patient would not receive the required care without their (the paediatrician's) involvement was rated as a very important barrier to discharge from the clinic by $49 \%(n=36)$ of the sample. The next most highly rated barrier was that it is too complicated to arrange discharge; rated as a very important influence by one-third of paediatricians $(33 \%, n=24)$. Over $60 \%$ of respondents indicated that parents being resistant to discharge is at least a somewhat important influence on their decision to discharge $(64 \% ; n=47)$. The majority of paediatricians indicated feeling pressure to discharge patients due to long clinic waiting lists as at least a somewhat important influence $(63 \%, n=45)$. Nearly one-third (30\%, $n=22)$ of respondents indicated having a good rapport with patients as being at least a somewhat important influence on their decision to discharge (Table 2).

Few characteristics of the paediatricians themselves were associated with their decisions to discharge a patient. Compared to males, more females stated that parents' resistance to their child being discharged from the clinic would be a somewhat important 
or very important influence on their discharge decisions (48 $v$. $78 \% ; P=0.01)$. We investigated differences between type of speciality and the importance of the pressure to discharge patients due to long waiting lists. We observed that general paediatricians more often stated this pressure as at least somewhat important compared to other specialist paediatricians $(77 v .51 \% ; P=0.02)$. No other responses to items differed significantly between general and specialist paediatricians.

\section{Parental influence on the follow-up and discharge process}

We asked paediatricians how often, as a proportion of patients over the past 12 months, parents had influenced their follow-up and discharge decisions. In total, 14\% $(n=10)$ of paediatricians reported parental pressure had frequently influenced how soon they would follow up with a child, with $53 \%(n=39)$ responding that this sometimes had an influence, and one-third $(33 \%, n=24)$

Table 1. Participant characteristics

\begin{tabular}{ll}
\hline Characteristic & $\%(n)$ \\
\hline Gender & \\
$\quad$ Female & $55(41)$ \\
Speciality & \\
$\quad$ Developmental & $16(12)$ \\
Endocrinology & $11(8)$ \\
General & $43(32)$ \\
Neurology & $16(12)$ \\
Respiratory & $14(10)$ \\
Years of specialist experience & \\
$\quad<5$ & $15(11)$ \\
$5-10$ & $22(16)$ \\
$11-20$ & $34(25)$ \\
$>20$ & $30(22)$ \\
Proportion of full-time equivalent worked in specialist clinic & \\
$0.1-0.4$ & $55(41)$ \\
$0.5-0.9$ & $24(18)$ \\
1 & $20(15)$ \\
\hline
\end{tabular}

stating parents rarely influenced their decisions. Of all 74 paediatricians, $57 \%(n=42)$ stated that parents asking to book another appointment 'just in case' would at least sometimes influence their decision to discharge the patient back to their GP (Table 3). No associations between paediatricians' characteristics and parental involvement in follow-up or discharge were observed.

\section{Discussion}

The most important finding from our study was that the factor influencing the greatest proportion of paediatricians in their decision to discharge patients back to primary care was the potential that patients may not receive the required care from a GP. Recent data show that GPs in Australia are seeing a smaller proportion of paediatric patients overall and a low number of extended consultations for children (Freed et al. 2013). As such, some may feel uncomfortable caring for a child with a chronic condition (Freed et al. 2016). This diminished experience of GPs in the care of children may indirectly affect the discharge behaviours of paediatricians, making them reluctant to relinquish the care of children back to primary care. One further aspect of hesitancy for discharge may be that other required speciality services in the community may not be perceived as being of the same quality as those found in tertiary settings.

In all, $70 \%$ of paediatricians in our study indicated the process of discharge being too complicated at least sometimes influenced their decision to send patients back to their GP. Although no recent data exist on this topic, one study from 1997 found that specialists perceived the increased workload of the discharge process as a barrier (Burkey et al. 1997). In particular, physicians in their study mentioned the added time required to explain discharge plans to patients, and to prepare related paperwork.

Strategies to improve discharge processes and support the transitions from paediatricians to GPs may increase the likelihood of patients being discharged. Others have suggested strategies such as making discharge consultations high priority, and allowing

Table 2. Factors influencing paediatricians' decisions to discharge patients from their clinic to the patient's GP $(n=73)$

\begin{tabular}{|c|c|c|c|c|}
\hline & $\begin{array}{c}\text { Unimportant, } \\
\%(n)\end{array}$ & $\begin{array}{c}\text { Not very } \\
\text { important, } \\
\%(n)\end{array}$ & $\begin{array}{c}\text { Somewhat } \\
\text { important, } \\
\%(n)\end{array}$ & $\begin{array}{c}\text { Very } \\
\text { important, } \\
\%(n)\end{array}$ \\
\hline Concerned patient may not receive required care without specialist involvement & $1(1)$ & $8(6)$ & $41(30)$ & $49(36)$ \\
\hline Too complicated to arrange for a GP to take over the care of a patient & $8(6)$ & $22(16)$ & $37(27)$ & $33(24)$ \\
\hline Parents are resistant to their child being discharged from the clinic & $0(0)$ & $36(26)$ & $56(41)$ & $8(6)$ \\
\hline Pressure to discharge patients because the clinic waiting list is very long $(n=72)$ & $14(10)$ & $24(17)$ & $46(33)$ & $17(12)$ \\
\hline Paediatrician has a good rapport with a patient and looks forward to seeing them again & $26(19)$ & $44(32)$ & $27(20)$ & $3(2)$ \\
\hline
\end{tabular}

Table 3. Frequency of parents influencing the follow-up and discharge process $(n=73)$

\begin{tabular}{|c|c|c|c|c|}
\hline & $\begin{array}{l}\text { Rarely, } \\
\%(n)\end{array}$ & $\begin{array}{l}\text { Sometimes, } \\
\%(n)\end{array}$ & $\begin{array}{l}\text { Frequently, } \\
\%(n)\end{array}$ & $\begin{array}{c}\text { Almost } \\
\text { always, } \\
\%(n)\end{array}$ \\
\hline Parental pressure influenced how soon you would follow up with a child & $33(24)$ & $53(39)$ & $14(10)$ & $0(0)$ \\
\hline $\begin{array}{l}\text { Parents asked to book another appointment 'just in case' even though you } \\
\text { were ready to discharge the patient }\end{array}$ & $43(31)$ & $44(32)$ & $11(8)$ & $3(2)$ \\
\hline
\end{tabular}


sufficient time to arrange the process of discharge (Burkey et al. 1997). These authors also suggest use of structured discharge criteria, which may reduce paediatricians' workload and associated complications surrounding the discharge process (Burkey et al. 1997). Standardisation and use of discharge templates has been suggested by the Department of Health, Victoria, as a strategy to streamline discharge processes, though no research to date has investigated whether this suggestion have been implemented (Department of Health and Human Services Victoria Australia 2010).

We asked paediatricians about parental influence on their decisions about discharge to GPs and determining time between follow-up appointments. Over $60 \%$ of paediatricians indicated they would be influenced by parents who are resistant to discharge their child back to their GP. We found that $67 \%$ of paediatricians would at least sometimes be influenced by parental pressure when determining time between specialist appointments. If parents are requesting to return to paediatricians earlier than required, this can unnecessarily affect the new-to-review visit ratio, with review patients using more appointment slots.

At the primary-speciality care interface, the medical home concept relies on paediatricians to discharge patients back to their GP when appropriate, and for GPs to accept the burden of care coordination for the child. Previous Australian research shows that over one-quarter of GPs sometimes, frequently or almost always have patients who don't return after referral to an outpatient paediatrician (Turbitt et al. 2016). Research from the UK suggests that inadequate communication between GPs and specialists can result in duplication of review of patients by both GPs and specialists, providing further rationale for the importance of a system supporting coordination of care (Hughes et al. 2003).

The Victorian Department of Health's 2013 Specialist Clinics Service Improvement Guide recommends strengthening links between specialist outpatient clinics and community health services such as GPs to improve discharge processes (Department of Health and Human Services Victoria Australia 2013). Suggestions include helping patients find a local GP if they do not have one, for specialist clinic staff to provide support and education to GPs, and for specialists to be receptive to queries from GPs. Future research could investigate whether these recommendations have been implemented and to what effect.

This study had some limitations. The sample was restricted to four chosen subspecialty and general paediatricians in Victoria; thus, results may not be generalisable to other specialties or states or countries. As we relied on paediatrician report rather than studying actual practice, our results may be subject to recall bias. Our findings are limited to the responses to items contained in our questionnaire and it is possible that additional factors influence discharge behaviours, which were not studied here.

\section{Conclusion}

Paediatricians may be reluctant to discharge patients from outpatient clinics if they believe follow-up care from a GP may be inadequate, or if discharge processes are too complicated. Improvements to the discharge process may encourage more paediatricians to discharge patients back to their GP, therefore freeing up appointment slots. This in turn could reduce waiting times for paediatric outpatient clinics in Victoria.

\section{Conflicts of interest}

The authors have no conflicts to declare.

\section{Acknowledgements}

This study was supported by funding from The Royal Children's Hospital Foundation and State of Victoria Department of Health.

\section{References}

American Academy of Paediatrics (2002) The medical home. Pediatrics 110, 184-186. doi:10.1542/peds.110.1.184

Burkey Y, Black M, Reeve H, Roland M (1997) Long-term follow-up in outpatient clinics. 2. The view from the specialist clinic. Family Practice 14, 29-33. doi:10.1093/fampra/14.1.29

Department of Health and Human Services Victoria Australia (2010) Victorian public hospital specialist clinics: discharge guidelines. Available at https://www2.health.vic.gov.au/Api/downloadmedia/\% 7B8F65DC06-ADAB-4331-9E8A-E8F8F0814788\%7D [Verified 15 October 2016]

Department of Health and Human Services Victoria Australia (2013) Specialist clinics service improvement guide. Available at https://www2. health.vic.gov.au/Api/downloadmedia/\%7B8946077D-7254-493F-B5C 7-7886DD5ACE16\%7D [Verified July 15 October 2016]

Dodd KL, Rhead S, Towey K (1994) Paediatric medical outpatients: are all those reviews necessary? Archives of Disease in Childhood 70, 493-495. doi:10.1136/adc.70.6.493

Freed GL, Spike NA, Sewell JR, Moran LM, Britt H, Valenti L, Brooks P (2013) Changes in longer consultations for children in general practice. Journal of Paediatrics and Child Health 49, 325-329. doi:10.1111/ jpc. 12157

Freed GL, Turbitt E, Kunin M, Gafforini S, Sanci L, Spike NA (2016) General practitioner perspectives on referrals to paediatric public specialty clinics. Australian Family Physician 45, 747-753.

Hughes ML, Leslie SJ, McInnes GK, McCormac K, Peden NR (2003) Can we see more outpatients without more doctors? Journal of the Royal Society of Medicine 96, 333-337. doi:10.1258/jrsm.96.7.333

Kunin M, Turbitt E, Gafforini S, Sanci L, Spike N, Freed G (2016) Parental preferences for paediatric specialty follow-up care. Australian Health Review, in press. doi:10.1071/AH16062

National Health and Hospitals Reform Commission (2009) A healthier future for all Australians: final report June 2009. Available at http://www.health. gov.au/internet/nhhrc/publishing.nsf/Content/nhhrc-report[Verified July 2016]

Turbitt E, Kunin M, Gafforini S, Sanci L, Spike NA, Freed GL (2016) Perspectives of Australian general practitioners on shared care for paediatric patients. Australian Journal of Primary Health, in press. doi:10.1071/PY16002

Department of Health and Human Services Victoria Australia (2009) Victorian public hospital specialist clinics: strategic framework. Available at https://www2.health.vic.gov.au/Api/downloadmedia/\%7B4CC7D85 B-9488-498F-8A8D-AC650FC9DB4B\%7D [Verified 15 October 2016] 